\title{
L'institutionnalisation des camps de détention de musulmans ouïghours en Chine
}

Romain Micalef

\section{(2) OpenEdition}

\section{Journals}

Édition électronique

URL : https://journals.openedition.org/rdr/1251

DOI : $10.4000 /$ rdr. 1251

ISSN : 2534-7462

Éditeur

Presses universitaires de Strasbourg

\section{Édition imprimée}

Date de publication : 16 novembre 2020

Pagination : 141-156

ISBN : 979-10-344-0073-7

ISSN : 2493-8637

Référence électronique

Romain Micalef, "L'institutionnalisation des camps de détention de musulmans ouïghours en Chine », Revue du droit des religions [En ligne], 10 | 2020, mis en ligne le 16 novembre 2020, consulté le 02 mai 2022. URL : http://journals.openedition.org/rdr/1251 ; DOI : https://doi.org/10.4000/rdr.1251

\section{(c) (7) \&}

La revue du droit des religions est mise à disposition selon les termes de la Creative Commons Attribution - Pas d'Utilisation Commerciale 4.0 International - CC BY-NC 4.0. 


\section{L'INSTITUTIONNALISATION DESCAMPS DEDÉTENTION DEMUSULMANSOUÏGHOURS ENCHINE}

\section{Romain MICALEF}

Docteur en droit public, Aix-Marseille Université

\section{RÉSUMÉ}

Une série de circulaires et de directives du gouvernement chinois a été rendue publique le 24 novembre 2019 par le Consortium international des journalistes d'investigation (CIJI). Ce qu'il convient désormais d'appeler l'affaire des China cables fait état de la création et du fonctionnement de camps de détention de musulmans ouighours dans la province du Xinjiang en Chine. L'institutionnalisation de ces camps interroge quant à la qualification juridique d'une telle action du gouvernement chinois. Entre épuration ethnique, religieuse et crime contre l'humanité, le vocable divise et la communauté internationale peine à les dénoncer d'une même voix. Les institutions internationales comme l'ONU et la CPI restent - pour l'instant - en retrait sur ce sujet.

\section{Abstract}

A series of circulars and directives from the Chinese government were issued on 24 November 2019 by the International Consortium of Investigative Journalists (ICIJ). What is now known as the China cables affair relates to the establishment and operation of detention camps for Uighur Muslims in Xinjiang province in China. The institutionalization of these camps raises questions as to the legal characterization of such action by the Chinese government. Ethnic and religious cleansing and crimes against humanity are divisive terms and the international community is struggling to denounce them with one voice. International institutions such as the UN and the ICC remain - for the time being - in the background on this issue. 
T e 24 novembre 2019, plusieurs documents de l'administration centrale _chinoise étaient rendus publics par le Consortium international des journalistes d'investigation (CIJI). Il s'agit de circulaires et directives relatives à la création et au fonctionnement de camps de détention de musulmans ouighours dans la province du Xinjiang en Chine. Appelés tantôt «camps de formation » par le gouvernement chinois, tantôt «camps d'internement » par l'Union européenne, le vocable divise et le choix du terme approprié varie selon les points de vue. Si certains témoignages évoquaient déjà de tels camps depuis 2017, aucun document officiel n'en avait jusque-là prouvé l'existence.

Les autorités chinoises ont d'abord nié l'existence de ces camps ${ }^{1}$. En août 2018, lors d'une audition devant Gay McDougall, vice-présidente du Comité pour l'élimination de la discrimination raciale (CERD) des Nations unies, la délégation chinoise a démenti l'existence de camps de « rééducation », tout en admettant la mise en place de «centres de formation professionnelle» et d'autres «mesures» visant à lutter contre l'« extrémisme ${ }^{2} »$. Entendue à nouveau en novembre 2018 dans le cadre de l'examen périodique universel du Conseil des droits de l'homme des Nations unies, la délégation chinoise a dénoncé les «accusations politiques de quelques pays», et répété que les camps dispensaient une formation professionnelle destinée à combattre l'extrémisme ${ }^{3}$.

À l'étranger, certaines informations concernant l'existence de ces camps ont fuité dès $2017^{4}$. Cependant, dans un entretien accordé à l'agence de presse officielle Chine nouvelle en octobre 2018, Shohrat Zakir, le président

1. Un média d'État a fait brièvement mention du fait que 460000 Ouighours musulmans du sud du Xinjiang avaient été "transférés» en vue d'occuper des «emplois» ailleurs dans la région. Aucune information supplémentaire n'a été communiquée à ce propos et cette explication semble avoir été abandonnée.

2. Sous-entendu ici de nature religieuse.

3. Le gouvernement chinois donne à nouveau cette explication dans le livre blanc qu'il a publié le 18 mars 2019 sur la lutte contre le terrorisme et l'extrémisme et les droits de l'homme au Xinjiang: The Fight Against Terrorism and Extremism and Human Rights Protection in Xinjiang: http://english.www.gov.cn/archive/white_paper/2019/03/18/content_281476567813306.htm [consulté le 17 juin 2020].

4. Des Ouighours de la diaspora s'inquiétaient de ne plus avoir de nouvelles de leurs proches et de leurs amis restés au pays. Et des étudiants ouighours, retournés de gré ou de force en Chine après des études à l'étranger, avaient disparu à leur arrivée sur le territoire. Quand ils parvenaient à obtenir quelques informations, les Ouighours de l'étranger apprenaient que la police avait embarqué leurs proches et leurs amis en camp de rééducation: "parti étudier» est l'euphémisme prudent utilisé sur WeChat, l'application de messagerie chinoise étroitement surveillée. 
de la région autonome ouighoure du Xinjiang, minimisait ces témoignages et indiquait:

«Les personnes envoyées dans ces centres sont là pour apprendre le mandarin, acquérir des notions de science et améliorer leur connaissance de l'histoire et de la culture chinoises [...], suivre des cours de droit, notamment sur la Constitution, le Code pénal et la législation anti-extrémisme du Xinjiang, et acquérir au moins une compétence professionnelle [...] afin de répondre aux besoins du marché du travail.»

Une étude du chercheur allemand Adrian $Z e n z^{5}$ a confirmé l'ampleur du phénomène en fournissant une estimation du nombre de personnes internées. De plus, il recense 78 appels d'offres concernant la construction, l'extension ou le réaménagement de camps de détention (certains dépasseraient 10000 mètres carrés et l'un approchait les 80000 mètres carrés ${ }^{6}$ ). En confrontant les informations issues de documents et de déclarations des autorités locales avec des données démographiques, Adrian Zenz et d'autres chercheurs arrivent à la conclusion que plusieurs centaines de milliers de personnes, voire plus d'un million, sont internées dans des camps. Ces estimations ne tiennent pas compte du rapide accroissement de la population carcérale: selon les chiffres officiels, le nombre d'arrestations au Xinjiang est passé de 27000 à 227000 entre 2016 et 2017. Il équivaut à $21 \%$ du nombre total d'arrestations en Chine en 2017, alors que les habitants du Xinjiang ne représentent que $1,5 \%$ de la population chinoise ${ }^{7}$. On considère qu'à ce jour plus de $10 \%$ de la population musulmane adulte du Xinjiang a été incarcérée.

L'institutionnalisation de ces camps de détention s'inscrit-elle dans un processus plus large de légitimation d'une épuration religieuse des Ouighours musulmans souhaitée par le gouvernement chinois?

Il conviendra tout d'abord de présenter la manière dont le gouvernement chinois justifie la création de ces « camps de formation» (1), avant d'observer

5. Adrian Zenz, maître de conférences en méthodes de recherche sociale à l'École européenne de culture et de théologie, basée en Allemagne, est un expert des questions relatives à l'incarcération de masse des Ouighours dans les camps d'internement de la région autonome ouighoure du Xinjiang, dans le nord-ouest de la Chine.

6. Ces éléments écrits sont corroborés par des images satellites des sites: beaucoup d'entre elles ont d'abord été compilées à partir de Google Earth par Shawn Zhang, un étudiant en droit à l'Université de Colombie-Britannique, au Canada, puis le corpus s'est étoffé avec le concours de sociétés d'analyse d'images travaillant pour la BBC et d'autres médias.

7. J. Millwar, « Pourquoi la Chine veut rééduquer ses musulmans?», Books, nº 97, mai 2019. 
et d'analyser les différentes formes de dénonciations d'une telle institutionnalisation par la communauté internationale (2).

\section{LA JUSTIFICATION DE LA CRÉATION DE «CENTRES DE FORMATION » PAR LE GOUVERNEMENT CHINOIS}

La légitimation de la création de camps de détention de musulmans ouighours par les circulaires et directives du gouvernement chinois (1.2) repose essentiellement sur l'argument de la lutte contre le terrorisme et la radicalisation islamiste (1.1).

\subsection{L'ARGUMENT DE LA LUTTE CONTRE LA RADICALISATION ISLAMISTE}

La recherche scientifique portant sur la naissance et le développement de l'islam en Chine n'est pas nouvelle ${ }^{8}$. En 1913, Henri Cordier y consacrait un article dans la revue Journal des savants ${ }^{9}$ dans lequel on pouvait lire qu'un «certain Hadji Mahomed Ali, d'origine arabe, né dans l'île de Hainan, rattache au Yunnan l'expansion des musulmans en Chine ${ }^{10}$ ». En effet, tous les musulmans de Chine ne sont pas des Ouighours, en revanche la majorité des Ouighours sont de confession musulmane ${ }^{11}$. Les Chinois musulmans n'appartenant pas à l'ethnie ouighoure sont principalement désignés par le terme chinois «Hui». Bien que cette présente recherche cible uniquement la communauté musulmane des Ouighours ${ }^{12}$, il est important de souligner que les persécutions des musulmans dans l'histoire de la Chine font fi des distinctions ethniques. Elisabeth Allès écrivait à ce propos que "provocations antireligieuses et attitudes anticléricales ont profondément marqué l'histoire des Chinois musulmans (Hui) tout au long des $\operatorname{XIX}^{e}$ et XIX $^{e}$ siècles ${ }^{13} »$.

8. Nous entendons ici la recherche scientifique en sciences sociales au sens large, pouvant inclure des analyses juridiques sans y être exclusivement consacrée.

9. H. CoRDiER, «L'Islam en Chine», Journal des savants, févr. 1913, p. 56-67.

10. Ibid.

11. I. Beller-Hann, «Dru C. Gladney, Dislocating China. Muslims, Minorities, and Other Subaltern Subjects», Perspectives chinoises, no 91, sept.-oct. 2005, p. 61-63.

12. Les populations musulmanes du Xinjiang ont comme point commun de pratiquer un islam sunnite de rite hanéfite marqué par le soufisme. Seuls les Tadjiks du Pamir chinois dérogent à cette règle. Parlant des langues iraniennes du Pamir, ils se rattachent à la branche ismaélienne du chiisme.

13. E. AllĖs, «À propos de l'islam en Chine: provocations antireligieuses et attitudes anticléricales du XIXe siècle à nos jours», Extrême-Occident, n 24, 2002, p. 65-75. 
Cependant, comme le précise le même auteur, des distinctions sont faites par les Chinois musulmans eux-mêmes entre Ouighours et Hui ${ }^{14}$. D'ailleurs, si les Hui et les Ouighours sont les minorités musulmanes les plus visibles en Chine, elles ne sont cependant pas les seules. À leurs côtés sont aussi présents des Kazakhs, des Kirghiz, des Ouzbeks, des Salars et des Tatars (tous de souche turque), ainsi que des Tadjiks (de souche persane), des Dongxiang et des Bonan (de souche mongole) ${ }^{15}$. Peuplé majoritairement d'Ouighours, le Xinjiang (Turkestan oriental) constitue après l'Ouzbékistan le second foyer de peuplement turcophone musulman en Asie centrale ${ }^{16}$. Tardivement intégré à l'Empire chinois, il a connu un passé tumultueux fait d'ingérences extérieures et d'insurrections séparatistes ${ }^{17}$. Si le problème ouighour était jusqu'il y a peu moins connu que le problème tibétain, il n'en constitue pas moins aux yeux de Pékin une question bien plus aiguë ${ }^{18}$. En 2001, le gouvernement chinois a publié une série de documents présentant les musulmans ouighours comme une force terroriste exogène liée aux réseaux djihadistes transnationaux ${ }^{19}$.

Avec la série d'attentats des 30 et 31 juillet 2011 à $\operatorname{Kachgar}^{20}$ dans la province chinoise du Xinjiang à la frontière immédiate avec le Kazakhstan,

14. E. Allès, L. Chérif-Chebbi, C.-H. Halfon, «L'islam chinois, unité et fragmentation», Archives de sciences sociales des religions, $\mathrm{n}^{\circ} 115,2001, \mathrm{p} .15-47$.

15. M. Graziano, «Zhongguo: la Chine au centre du monde, même pour le Saint-Siège», Outre-Terre, $\mathrm{n}^{\circ}$ 15, 2006, p. 19-41.

16. Depuis sa conquête par les Qing, la région est appelée «Xinjiang» c'est-à-dire «nouvelle marche» ou «nouvelle frontière». Cependant, depuis le $\mathrm{xx}^{\mathrm{e}}$ siècle, la mouvance anticoloniale appelle la région «Turkestan oriental» (Shärkiy Türkistan en ouïghour) pour signifier son appartenance au monde turc et non au monde chinois. L'emploi inconsidéré de cette appellation (Dong Tujuesitan ou Dongtu en chinois) est assimilé par les autorités chinoises à des velléités séparatistes et peut conduire à de graves sanctions. Par analogie avec les ex-républiques soviétiques turcophones d'Asie centrale aujourd'hui indépendantes, une partie des militants nationalistes ouighours tend aussi à promouvoir l'emploi du terme Uyghuristan (pays des Ouighours).

17. A. Kumul, "Le séparatisme ouïghour au xxe siècle: Histoire et actualité », Cahiers d'études sur la Méditerranée orientale et le monde turco-iranien, $\mathrm{n}^{\circ}$ 25, 1998.

18. M. Rollinde (dir.), Les droits culturels: Enjeux et contradictions, Paris, CEDIDELP, déc. 2005.

19. R. CASTETS, «Opposition politique, nationalisme et islam chez les Ouighours du Xinjiang», Les études du CERI, nº 110, oct. 2004.

20. Le massacre de 2011 à Kachgar est une série d'attaques à la bombe et à l'arme blanche entre le 30 et le 31 juillet 2011. Le 30 juillet, deux Ouighours s'emparent d'une camionnette, tuant le conducteur, et roulent vers les piétons. Les deux hommes descendent de la camionnette et poignardent six individus à mort et en blessent vingt-sept autres. L'un des deux assaillants est tué par la foule; l'autre est appréhendé. Le 31 juillet, deux explosions retentissent dans un restaurant. Un groupe d'Ouighours armés tue deux personnes à l'intérieur du restaurant ainsi que quatre autres à l'extérieur, blessant quinze autres 
ainsi qu'avec l'attentat du 22 mai 2014 dans un marché d'Urumqi (capitale du Xinjiang) qui a fait 31 morts et 90 blessés $^{21}$, le gouvernement chinois fit un lien direct entre ces événements et un développement de l'idéologie djihadiste dans la région. Ces attentats marquent un tournant dans l'intensification des persécutions des musulmans ouighours sous couvert d'une lutte contre la radicalisation islamiste et le terrorisme djihadiste. La construction de camps de détention est une des conséquences directe et majeure de la volonté de Pékin de gommer tout particularisme de cette ethnie principalement marquée par son appartenance religieuse. Des clichés photographiques, ainsi que des articles de presse datant de 2017 ont mis en évidence le caractère punitif de ces centres construits dans le désert, avec de hauts murs d'enceinte, des barbelés et des miradors. Des témoignages d'anciens détenus ont été recueillis par Foreign Policy et The Washington Post. Ils permettent de se faire une idée de la manière dont ce type de camp fonctionne: les internés sont contraints de chanter des chansons communistes, de renier l'islam, de faire leur autocritique et de dénigrer les croyances de leur famille, de regarder des films de propagande, d'étudier le mandarin et l'histoire de la Chine. Les témoignages concordent, présentant des cellules surpeuplées et des rations de nourriture restreintes et infectes. Il en ressort aussi que les détenus qui se plaignent sont «placés à l'isolement, privés de repas, menottés au mur ou attachés aux poignets et aux chevilles sur une "chaise du tigre", voire soumis à des séances d'électrochocs ou au simulacre de la noyade $22 »$. Ce type de pratiques et de tortures n'est pas sans rappeler le camp de Guantánamo. En effet, dans ce centre de détention militaire de haute sécurité, situé sur la base navale américaine de Guantánamo, dans le sud-est de Cuba, sont détenues des personnes qualifiées de «combattants illégaux », capturées par l'armée américaine dans les différentes opérations qu'elle mène à l'étranger contre des militants et terroristes islamistes. En juin 2006, la Cour suprême des États-Unis a estimé, dans l'arrêt Hamdan $v$. Rumsfeld, que ces procédures judiciaires d'exception étaient illégales et violaient les conventions de Genève ${ }^{23}$.

victimes. La police abat quatre des assaillants et appréhende le reste du groupe. Le gouvernement chinois affirme que les auteurs prônent le djihad et que ceux-ci font partie du Parti islamique du Turkestan (MITO).

21. "Chine: plus de 30 morts dans un attentat au Xinjiang», Le Monde.fr: https://www. lemonde.fr/asie-pacifique/article/2014/05/22/chine-nouvel-attentat-meurtrier-a-urumqicapitale-du-xinjiang_4423326_3216.html [consulté le 17 juin 2020].

22. J. Millwar, «Pourquoi la Chine veut rééduquer ses musulmans?», art. cit.

23. Hamdan v. Rumsfeld, 548 U.S. 557 (2006). 
Plusieurs condamnations pour prosélytisme religieux ont été prononcées. Il s'agit d'infractions qualifiées de crimes donnant lieu à un jugement souvent peu motivé. Le 12 juin 2018, la cour criminelle de la région autonome du Xinjiang a rendu un arrêt - traduit en anglais - condamnant un homme ouïghour musulman pour prosélytisme ${ }^{24}$. Il est accusé « d'incitation à la haine et à la discrimination ethnique et religieuse» envers le peuple chinois. L'arrêt rapporte qu'il aurait tenu des propos religieux extrémistes. Selon l'arrêt, le défendant aurait tenu les propos suivants:

«Ne dites pas de gros mots, ne regardez pas de porno ou vous deviendrez un kafir (non-croyant). Si vous ne priez pas, votre âme ne sera pas nettoyée pendant 40 jours et Dieu n'acceptera pas vos prochaines prières. Si vous mangez sans prier, vous deviendrez un kafir. Si vous ne priez pas, vous irez en enfer et Dieu ne vous pardonnera pas. Toutes les personnes qui ne prient pas sont des kafirs chinois Han. Vous ne pouvez pas manger de la nourriture de femmes qui ne prient pas. Vous ne pouvez pas manger la nourriture des personnes qui fument et boivent de l'alcool».

Le défendant a été condamné de manière lapidaire, avec une décision de justice sommaire, pour le crime "d'incitation à la haine et discrimination ethnique et religieuse» à dix ans de prison et à la privation de droits politiques pendant cinq ans ${ }^{25}$.

C'est dans cette même dynamique, que le Bureau de l'information du Conseil des affaires d'État (gouvernement chinois) a publié, le 18 mars 2019, un livre blanc relatif à la lutte contre le terrorisme et l'extrémisme et sur la protection des droits de l'homme au Xinjiang ${ }^{26}$. Le document dispose que «c'est seulement en renforçant notre communauté de destin, en abandonnant le deux poids deux mesures, en intensifiant la confiance politique mutuelle, en atteignant le consensus stratégique et en promouvant les échanges et la coopération, que nous pouvons effectivement freiner et combattre le terrorisme et l'extrémisme dans l'intérêt de la paix et de la stabilité du monde ${ }^{27} »$. En plus d'une préface et d'une conclusion, le livre blanc se compose de sept sections principales: «Le Xinjiang est depuis longtemps une partie

24. Xinjiang Uighur Autonomis Region Qakilik Country People's Court, Criminal Judgement, June 12, 2018: https://www.icij.org/investigations/china-cables/read-the-china-cablesdocuments/ [consulté le 27 mai 2020].

25. «For the crime of inciting ethnic hatred and ethnic discrimination, is sentenced to prison for ten years and the deprivation of political rights for five years»: ibid.

26. The Fight Against Terrorism and Extremism and Human Rights Protection in Xinjiang, op. cit.

27. Ibid. 
inséparable du territoire chinois », «L'origine du terrorisme et de l'extrémisme au Xinjiang», «Le terrorisme et l'extrémisme religieux violent constituent de graves atteintes aux droits de l'homme», "Lutter contre le terrorisme et l'extrémisme conformément à la loi », «Accorder la priorité absolue à une approche préventive dans la lutte contre le terrorisme», "Trouver de l'expérience pour lutter contre le terrorisme et la déradicalisation », ainsi que «La coopération et les échanges internationaux en matière de lutte contre le terrorisme ${ }^{28} »$.

\subsection{LES DIRECTIVES ET CIRCULAIRES DE L'ÉTAT RECONNAISSANT LA CRÉATION ET LE FONCTIONNEMENT DES « CAMPS DE FORMATION »}

Les documents rendus publics le 24 novembre 2019 font état de quatre directives et d'une circulaire. Bien que la qualification juridique de ces documents édictés par l'administration centrale chinoise ne soit pas complètement assimilable aux catégories connues par le droit administratif français, il ne paraît pas excessif en l'espèce de retenir la dénomination de directives et de circulaires. Le droit administratif chinois connaît aussi ce type d'acte administratif permettant à l'administration centrale de donner des indications, précisions ou injonctions à des administrations locales déconcentrées. Il s'agit des actes administratifs ordinaires ${ }^{29}$. Ces actes administratifs sont aussi des vecteurs permettant de renforcer la tradition juridique marxiste que le gouvernement communiste chinois souhaite préserver et alimenter. À ce propos, certains auteurs parlent d'un «droit administratif instrumentalisé $^{30} »$.

Le premier document est une circulaire intitulée Autonomous Region State Organ Telegram. Il est daté de 2017 et porte le numéro 41931. Ce document fait état de la création de «centres de formation professionnelle» qu'il convient de nommer «centres de formation». La circulaire souligne la volonté du gouvernement chinois de lutter contre le terrorisme et de maintenir la stabilité politique de la région. Pour ce faire, elle précise qu’il convient de

28. Ibid.

29. L. ZHANG, «La procédure administrative en Chine», RID comp. 2012, p. 451-475.

30. L. ZHANG, «L'évolution du droit administratif chinois dans les trente dernières années (1978-2008)», Chaire M.A.D.P. de Sciences Po: https://www.sciencespo.fr/chaire-madp/ sites/sciencespo.fr.chaire-madp/files/zhang_li.pdf [consulté le 17 juin 2020].

31. Autonomous Region State Organ Telegram, $\mathrm{n}^{\circ} 419$, 2017, document disponible en langue chinoise et anglaise: https://www.icij.org/investigations/china-cables/read-the-china-cablesdocuments/ [consulté le 17 juin 2020]. 
renforcer et normaliser davantage le travail des «centres de formation» afin d'assurer la sécurité absolue du lieu de formation, d'améliorer la qualité et l'efficacité de l'éducation et de la formation, de maximiser l'éducation, et de promouvoir la stabilité sociale et la stabilité à long terme de l'ensemble du Xinjiang. La circulaire se divise en cinq parties. La première prend la forme d'une injonction et s'intitule «Tout d'abord, assurez-vous que le lieu de formation est absolument sûr ». Il y est expliqué comment se préparer et réagir aux éventuelles évasions, comment prévenir les éventuels troubles en privant les détenus de leurs téléphones cellulaires et la manière dont doivent se comporter les gardes, notamment en patrouillant 24 heures sur 24 et en n'hésitant pas à recourir à la force si nécessaire. La seconde partie concerne la manière de procéder afin d'améliorer la qualité de l'éducation et de l'entraînement des détenus. L'apprentissage du mandarin est imposé et le recrutement des professeurs de chinois est strictement encadré. Le texte contient aussi un paragraphe dédié à l'éducation idéologique (ideological education) et familiale (familial education). La troisième partie est dédiée au management de l'équipe éducative. Il est fait état de zones dans lesquelles le management doit être plus strict que dans d'autres zones (a strict management area). La quatrième partie concerne la mise en place d'examens d'évaluation et la distribution de points attribués notamment sur la réussite de la «transformation idéologique» (ideological transformation) du détenu. La cinquième et dernière partie concerne la préparation de la sortie du détenu afin qu'il trouve un travail et qu'il devienne un «bon citoyen» impliqué dans la société civile. La circulaire se termine sur l'importance de garder ce document secret et d'enjoindre les membres des équipes travaillant dans ces camps à la plus grande discrétion. La circulaire précise à ce titre: "Il est nécessaire de sensibiliser davantage le personnel au secret, à une discipline politique sérieuse et à la discipline du secret. Il est strictement interdit d'apporter des équipements vidéo tels que des téléphones portables et des caméras dans les zones d'enseignement et de gestion et de télécharger des photos sur Internet à volonté.»

Une directive du 16 juin 2017 intitulée Integrated Joint Operation Platform ${ }^{32}$ concerne la situation des Ouighours ayant obtenu un passeport étranger ou tout autre type de visa leur permettant de se rendre à l'étranger. Leur nombre est donné pour toutes les villes du Xinjiang. Il est expliqué qu'à l'avenir, en dehors de ceux vérifiés par les organes de sécurité publique, pour toute personne originaire du Xinjiang qui obtient la nationalité étrangère ou un

32. Integrated Joint Operation Platform, 16 juin 2017 : https://www.icij.org/investigations/chinacables/read-the-china-cables-documents/ [consulté le 17 juin 2020]. 
passeport étranger, ou qui demande un visa dans les ambassades ou consulats chinois à l'étranger, ou pour toute personne du Xinjiang qui demande le remplacement de documents valides dans les ambassades ou consulats chinois à l'étranger, le comité politique et juridique devra utilisé la plateforme «intégrée» et «pour ceux dont le terrorisme ne peut être exclu, un contrôle aux frontières devra être mis en place pour procéder à l'arrestation ou pour refuser l'approbation». Les trois autres directives du 21, 25 et 29 juin 2019 fournissent des éléments permettant l'identification des musulmans ouighours chinois et étrangers et les procédures à suivre afin de les interner dans ces «camps de formation ${ }^{33}$. Récemment, la Cour européenne des droits de l'homme s'est prononcée sur l'expulsion d'Ouighours vers la Chine par la Bulgarie. La Cour considère que si une telle expulsion avait lieu, cela constituerait une violation des articles 2 et 3 de la Convention (CEDH, 20 févr. 2020, M.A. et a. c. Bulgarie).

Le professeur de droit Zhang $\mathrm{Li}^{34}$ explique que «pendant les dix dernières années, pour promouvoir son ambition de créer une "société harmonieuse", la Chine a pris des mesures dans le domaine du contrôle de l'Administration. Cependant, à défaut de considérations approfondies, ce processus [...] a généré des conséquences négatives sur l'affirmation du principe de l'État de droit en Chine ${ }^{35} »$. Le contrôle déconcentré de l'État chinois sur l'administration locale de la province du Xinjiang, par l'entremise de circulaires et directives légalisant les camps de détention de musulmans ouighours, risque d'élever une muraille entre «l'affirmation du principe de l'État de droit» et la création d'une «société harmonieuse », toutes deux souhaitées - a priori - par le gouvernement de la République populaire de Chine.

33. Directives des 21, 25 et 29 juin 2019: https://www.icij.org/investigations/china-cables/ read-the-china-cables-documents/ [consulté le 17 juin 2020].

34. Madame le Professeur Zhang Li est professeur de droit à l'Université de droit et de science politique de la Chine.

35. L. ZHANG, «Les mécanismes de contrôle de l'Administration en Chine. Une société harmonieuse peut-elle se construire au détriment de l'État de droit? », RF adm. publ. 2014, p. 371-392. 


\section{LA DÉNONCIATION PAR L'UNION EUROPÉENNE ET L'ORGANISATION DES NATIONS UNIES DE L'INSTITUTIONNALISATION DES « CAMPS D'INTERNEMENT »}

Si l'Union européenne s'est officiellement prononcée sur les circulaires et directives légalisant les camps de détention de musulmans ouïghours (2.1), l'ONU tarde à prendre position sur cette question alors qu'elle est pressée de le faire par de nombreux gouvernements étrangers (2.2).

\subsection{LA DEMANDE DE FERMETURE DES «CAMPS D'INTERNEMENT » D'OUÏGHOURS PAR L'UNION EUROPÉENNE}

Dans un communiqué de presse du 19 décembre $2019^{36}$, le Parlement européen déclare que

«la pression s'est accentuée sur Pékin après une récente fuite de documents classés (China cables). Ces derniers semblent confirmer les informations faisant état de la détention par les autorités chinoises de plus d'un million de musulmans, principalement ouighours, dans des "camps de rééducation" dans la région du Xinjiang (nord-ouest). Les autorités chinoises affirment que ces "centres de formation" sont utilisés pour lutter contre l'extrémisme religieux violent».

Les députés européens sont visiblement préoccupés par la répression chinoise des Ouighours et appellent le gouvernement chinois à fermer immédiatement les «camps de rééducation» dans le Xinjiang. Dans une résolution adoptée le 19 décembre $2019^{37}$, les députés européens condamnent fermement le fait que des centaines de milliers de musulmans ouighours soient envoyés dans des «camps d'internement»-nommés aussi par eux «camps de rééducation»-sur la base d'un système de «police préventive». Ils exhortent le gouvernement chinois à arrêter immédiatement la pratique des détentions arbitraires sans inculpation, jugement ou condamnation pour infraction pénale, et à libérer immédiatement et sans condition toutes les personnes détenues, y compris Ilham Tohti, lauréat du Prix Sakharov 2019. Cette résolution est juridiquement fondée sur plusieurs visas se référant aux

36. Communiqué de presse du Parlement européen: https://www.europarl.europa.eu/news/ fr/press-room/20191212IPR68927/la-chine-doit-fermer-ses-camps-de-reeducation-pourouighours [consulté le 17 juin 2020].

37. Résolution du Parlement européen du 19 décembre 2019 sur la situation des Ouighours en Chine (China Cables) (P9_TA(2019)0110). 
orientations de l'Union européenne relatives à la promotion et à la protection de la liberté de religion ou de conviction, adoptées par le Conseil «Affaires étrangères» le 24 juin 2013, ainsi qu'aux précédentes résolutions sur la situation en Chine, en particulier celles du 18 avril 2019 sur la situation des minorités religieuses et ethniques en Chine ${ }^{38}$, du 4 octobre 2018 sur la détention arbitraire de masse d'Ouighours et de Kazakhs dans la région autonome ouighoure du Xinjiang ${ }^{39}$, du 12 septembre 2018 sur l'état des relations entre l'Union européenne et la Chine ${ }^{40}$.

La résolution du 19 décembre 2019 dénonce une aggravation de la situation des musulmans du Xinjiang. Selon les eurodéputés, depuis l'arrivée au pouvoir du président Xi Jinping en mars 2013, le gouvernement chinois a accru son hostilité envers les libertés religieuses. Ils considèrent que les autorités chinoises mènent une campagne de plus en plus intense d'internement de masse, de surveillance numérique intrusive (notamment avec la technologie de reconnaissance faciale et la collecte de données), d'endoctrinement politique et d'assimilation culturelle forcée. Selon les eurodéputés toujours,

«les Ouighours et d'autres minorités ethniques principalement musulmanes dans la région autonome ouighoure du Xinjiang ont fait l'objet de détentions arbitraires, de tortures, de restrictions draconiennes pour ce qui est de la pratique et de la culture religieuses, et d'une surveillance numérique envahissante au point que tous les aspects de la vie sont contrôlés, au moyen de caméras de reconnaissance faciale, d'inspection du contenu de leurs téléphones mobiles, de prélèvements d'ADN et d'une présence policière étendue et intrusive ${ }^{41}$ ».

Il est indiqué qu'environ un million de personnes sont ou ont été détenues arbitrairement dans des centres dits "de rééducation politique» pour une durée indéterminée, sous prétexte de lutte contre le terrorisme et l'extrémisme religieux. In fine, la résolution précise qu'il s'agit de l'incarcération de masse la plus importante d'une minorité ethnique et religieuse à l'heure actuelle dans le monde ${ }^{42}$.

38. P8_TA(2019) 0422 .

39. P8_TA(2018)0377.

40. P8_TA(2018)0343.

41. Résolution, 19 déc. 2019, précit.

42. Les camps d'internement au Xinjiang ont connu une expansion rapide après la nomination de Chen Quango au poste de secrétaire du Parti communiste de la région en août 2016. Shohrat Zakir, président de la région du Xinjiang, a affirmé en décembre 2019 que les 1,5 million de personnes placées dans des camps de rééducation et d'internement avaient toutes été «rendues à la société», sans fournir aucune preuve. 
Inquiets face aux mesures répressives que subissent les Ouighours et les autres minorités ethniques musulmanes, les eurodéputés demandent dans cette résolution que les autorités chinoises respectent leurs libertés fondamentales. De plus, ils condamnent l'envoi de centaines de milliers d'Ouighours dans ces «camps de rééducation politique» sur la base d'un système de "police prédictive», y compris pour des motifs tels que des voyages à l'étranger ou une foi religieuse jugée trop ardente. Ils demandent au gouvernement chinois de mettre un terme à la pratique des détentions arbitraires de membres des minorités ouighoures, de fermer tous les camps et centres de détention et de libérer les détenus immédiatement et sans condition. Enfin, ils soulignent que «toute forme de détention, lorsqu'elle est appliquée en violation des lois internationales fondamentales, ainsi que la persécution à l'encontre de personnes ou de groupes donnés en raison de motifs ethniques, culturels ou religieux, ou d'autres actes inhumains causant de profondes souffrances ou de graves blessures aux populations civiles sont inacceptables à la lumière du cadre juridique international ${ }^{43} »$.

\subsection{L'OUVERTURE D'UNE ENQUÊTE DE L'ORGANISATION DES NATIONS UNIES SOUHAITÉE PAR LES GOUVERNEMENTS ÉTRANGERS}

En août 2018, le Comité des Nations unies pour l'élimination de la discrimination raciale a mis en cause le gouvernement de la République populaire de Chine en raison des abus commis au Xinjiang, notamment la création de camps de détention arbitraire de masse. En septembre 2018, lors de son tout premier discours après sa prise de fonction, Michelle Bachelet, Haut-Commissaire des Nations unies aux droits de l'homme, a pris acte des «allégations profondément préoccupantes d'arrestations arbitraires à très grande échelle d'Ouighours et de membres d'autres communautés musulmanes, internés dans des camps dits de rééducation dans tout le Xinjiang ${ }^{44} »$. Le gouvernement chinois a refusé les demandes de la Haut-Commissaire des Nations unies aux droits de l'homme en vue de l'envoi d'enquêteurs indépendants au Xinjiang et de leur accès aux camps.

Le 8 juillet 2019, le Conseil des droits de l'homme des Nations unies a publié une déclaration conjointe de vingt-deux États exhortant la Chine à

43. Résolution, 19 déc. 2019, précit.

44. Premier discours de Michelle Bachelet lors de sa prise de fonction de Haut-Commissaire des Nations unies aux droits de l'homme: https://www.rts.ch/info/monde/9832475-premier-discours-de-michelle-bachelet-au-conseil-des-droits-de-l-homme.html [consulté le 17 juin 2020]. 
mettre fin aux détentions arbitraires de masse et aux violations connexes contre les musulmans dans la région du Xinjiang ${ }^{45}$. Dans leur démarche sans précédent, ces pays ont également appelé la Chine à coopérer avec le Haut-Commissaire des Nations unies aux droits de l'homme et les experts de l'ONU pour permettre un accès significatif à la région. Ils se sont déclarés préoccupés par les informations faisant état de détentions arbitraires à grande échelle, d'une surveillance étendue et d'autres violations contre les Ouighours musulmans du Xinjiang.

Depuis cette date, l'ONU ne s'est toujours pas officiellement prononcée sur les circulaires et directives légalisant des camps d'internement de musulmans ouighours en Chine, mais certains éléments laissent à penser qu'elle devrait le faire dans un futur plus ou moins proche. En effet, le 29 octobre 2019, deux factions différentes ont soumis des déclarations à la session de la Troisième Commission sur le Comité pour l'élimination de la discrimination raciale (CERD) - un groupe critiquant les actions de la Chine et l'autre les soutenant, mais aucune résolution n'a été adoptée ${ }^{46}$.

Cependant, certains gouvernements enjoignent publiquement l'ONU d'ouvrir une enquête. Le Royaume-Uni a exhorté la Chine à donner aux observateurs des Nations unies "un accès immédiat et sans entraves» aux camps de détention du Xinjiang, où plus d'un million de personnes de la communauté ouighoure et d'autres minorités musulmanes sont détenues sans jugement ${ }^{47}$. Lors d'une conférence de presse le lundi 25 novembre 2019, le gouvernement allemand a condamné l'internement des Ouighours mais a insisté sur l'existence de pourparlers avec la Chine pour avoir accès aux camps et évaluer par lui-même la véracité des informations contenues dans lesdits documents ${ }^{48}$. Le 27 novembre 2019, la France a appelé la Chine à cesser ses «détentions arbitraires de masse» dans la région du Xinjiang, où plus d'un million de musulmans, principalement d'ethnie ouighoure, seraient détenus dans ce que Pékin présente comme des «centres de formation professionnelle ${ }^{49}$. Le ministre français des Affaires étrangères a déclaré le même jour devant la Commission des affaires étrangères de l'Assemblée nationale: « Nous exhortons

45. Déclaration du Conseil des droits de l'homme des Nations unies du 8 juillet 2019: https:// www.hrw.org/sites/default/files/supporting_resources/190708_joint_statement_xinjiang. pdf [consulté le 17 juin 2020].

46. V. à ce propos: https://thediplomat.com/2019/10/rival-camps-clash-over-xinjiang-at-uncommittee-session/ [consulté le 17 juin 2020].

47. V. à ce propos: https://www.theguardian.com/world/2019/nov/25/uk-calls-for-un-accesschinese-detention-camps-xinjiang [consulté le 17 juin 2020].

48. V. à ce propos: https://www.dw.com/en/china-uighurs-siemens-germanypartnership/a-51405883 [consulté le 17 juin 2020].

49. Discours de la porte-parole du Quai d'Orsay, 27 nov. 2019, source AFP. 
la Chine - outre à la fermeture des camps d'internement - à inviter dans les meilleurs délais la Haute-Commissaire aux droits de l'homme des Nations unies, Michelle Bachelet, et les experts des procédures spéciales, afin qu'ils rendent compte de manière impartiale de la situation ${ }^{50}$.»

Actuellement, l'initiative juridique la plus aboutie sur le plan international vient du Congrès des États-Unis d'Amérique qui a adopté le 3 décembre 2019 une version amendée du Uyghur Human Rights Policy Act du 17 janvier 201951, par lequel il presse le gouvernement fédéral des États-Unis de prendre des mesures immédiates afin de protéger les droits de l'homme et d'envisager d'imposer des sanctions économiques et en matière de visas, conformément à la loi Magnitsky, à l'encontre des fonctionnaires chinois dans la province du Xinjiang, tout en protégeant les Ouighours résidant aux États-Unis contre le harcèlement et les persécutions exercés par la Chine.

La Chine n'est pas partie aux statuts de la Cour pénale internationale (CPI). Ainsi - et cela est regrettable - le procureur de la CPI ne pourra pas ouvrir une enquête sur ces crimes présumés, comme il l'a fait - par exemple - dans le cas de la situation des Rohingyas en République populaire du Bangladesh. Cependant, le parallèle demeure intéressant concernant la détermination de la nature des crimes commis, bien que la compétence de la CPI soit exclue en l'espèce. En effet, le 14 novembre 2019, la Chambre préliminaire III $^{52}$ de la CPI a autorisé le procureur à procéder à une enquête sur les crimes présumés relevant de la compétence de la CPI qui auraient été commis en République de l'Union du Myanmar. Cette autorisation fait suite à la demande présentée le 4 juillet 2019 par le procureur d'ouvrir une enquête concernant des crimes qui auraient été commis contre le peuple rohingya du Myanmar ${ }^{53}$. Selon la Chambre, il existe de sérieux soupçons permettant de croire que des actes de violence généralisés et/ou systématiques pourraient être qualifiés de crimes contre l'humanité de déportation à la frontière entre le Myanmar et le Bangladesh et de persécution pour des motifs d'ordre ethnique et/ou religieux contre

50. Discours du ministre des Affaires étrangères devant la Commission des affaires étrangères de l'Assemblée nationale le 27 nov. 2019.

51. Version amendée le 3 déc. 2019 par le Congrès des États-Unis de Uighur Intervention and Global Humanitarian Unified Response Act (UIGHUR Act) du 17 janv. 2019.

52. La Chambre préliminaire III de la CPI est composée de la juge Olga Herrera Carbuccia, juge présidente, du juge Robert Fremr et du juge Geoffrey Henderson.

53. La Chambre a également reçu les vues de centaines de milliers de victimes présumées, ou en leur nom. Selon le greffe de la CPI, les victimes insistent à l'unanimité pour demander une enquête de la Cour et bon nombre des victimes présumées consultées «croient que seules la justice et la responsabilité peuvent garantir la fin du cercle perçu de violence et d'abus». La Chambre a reconnu toutes les personnes et organisations qui ont aidé, guidé et conseillé les victimes présumées tout au long de ce processus. 
la population rohingya ${ }^{54}$. En conséquence, la Chambre préliminaire III a autorisé l'ouverture de l'enquête pour tout crime, y compris les crimes futurs, tant que: a) il relève de la compétence de la Cour; b) il serait commis au moins en partie sur le territoire du Bangladesh ou le territoire de tout autre État partie ou État acceptant la compétence de la CPI; c) il est suffisamment lié à cette situation; et d) il aurait été commis après la date de l'entrée en vigueur du Statut de Rome pour le Bangladesh ou un autre État partie concerné $e^{55}$. Si le contexte chinois est différent, il n'en demeure pas moins qu'il s'agit dans les deux cas de persécutions religieuses envers des musulmans. Le parallèle reste donc pertinent, bien qu'une telle enquête pour crime contre l'humanité ne puisse s'ouvrir concernant le cas des Ouighours.

In fine, la communauté internationale tente de s'organiser au mieux afin de dénoncer d'une même voix la récente institutionnalisation des camps de détention de musulmans ouighours en Chine qui constitue un pas supplémentaire vers ce qui ressemble de plus en plus à une épuration ethnique et religieuse à grande échelle. Certains États restent tout de même en marge de ce processus de dénonciation. Il s'agit étonnamment de la majorité des États arabo-musulmans (intérêts économiques obligent), à l'exception de la Turquie qui semble se démarquer de cette réserve. Tous les regards sont désormais tournés vers l'ONU. Ses récentes et timides prises de position sur la question des persécutions des musulmans ouighours ne sont peut-être pas sans lien avec le statut de membre permanent que la Chine occupe au sein de son Conseil de sécurité. Le vote d'une nouvelle résolution mettrait un terme à cette suspicion et donnerait du corps à un engagement international relatif à la protection des droits de l'homme et de la liberté de religion, actuellement éparpillé entre le texte adopté par le Parlement européen le 19 décembre 2019 et les récentes prises de positions de certains gouvernements étrangers, diplomatiquement peu dissuasives et juridiquement non contraignantes.

54. Notant l'ampleur des crimes allégués et le nombre de victimes présumées, la Chambre a estimé que la situation atteignait clairement le seuil de gravité nécessaire pour l'ouverture d'une enquête de la Cour. Selon les documents fournis, entre 600000 et un million de Rohingyas auraient été déplacés de force du Myanmar vers le Bangladesh voisin à la suite d'actes de coercition allégués. Notant le point de vue des victimes, la Chambre était d'accord avec le procureur qu'il n'existait aucune raison sérieuse de croire qu'une enquête sur la situation ne serait pas dans l'intérêt de la justice.

55. Decision Pursuant to Article 15 of the Rome Statute on the Authorisation of an Investigation into the Situation in the People's Republic of Bangladesh/Republic of the Union of Myanmar. 